\title{
Optimal Harvest Control in a Singular Prey-Predator Fishery Model with Maturation Delay and Gestation Delay
}

\author{
Chao Liu, Longfei Yu, and Qingling Zhang \\ Institute of Systems Science, Northeastern University, Shenyang 110004, China \\ Correspondence should be addressed to Chao Liu; singularsystem@163.com
}

Received 18 August 2016; Accepted 20 October 2016

Academic Editor: Hubertus Von Bremen

Copyright (C) 2016 Chao Liu et al. This is an open access article distributed under the Creative Commons Attribution License, which permits unrestricted use, distribution, and reproduction in any medium, provided the original work is properly cited.

\begin{abstract}
This paper presents a singular prey-predator fishery model, where maturation delay for prey and gestation delay for predator are considered. Fishing efforts are introduced to harvest prey and predator population, which are developed as control instruments to investigate optimal utilization of fishery resource. By analyzing associated characteristic equation, local stability analysis is studied due to combined variations of double time delays. Furthermore, Pontryagin's maximum principle is utilized to characterize optimal harvest control, and the optimality system is numerically solved based on an iterative method.
\end{abstract}

\section{Introduction}

According to statistics from the Food and Agriculture Organization of United Nations [1], approximate fifty-three percent of fish stock under observation has experienced overexploitation or depletion, which reiterate the fact that fishery needs to be managed with an effective and carefully defined objective to prevent overexploitation and replenish depleted stock [1]. In order to ensure sustainable fishery, it requires precision in stock assessment and reliability in fishery modelling $[2,3]$. In recent decades, there are growing research interests in formulating bioeconomic mathematical models to obtain more comprehensive indications of feedback effect between exploitation activity and fishery resources [4-13].

In [5], authors establish a dynamic model where both prey and predator fishery resource population are exploitable, which is as follows:

$$
\begin{aligned}
& \dot{x}(t)=r x(t)\left(1-\frac{x(t)}{K}\right)-\frac{\beta x(t) y(t)}{\alpha+x(t)}-E(t) x(t), \\
& \dot{y}(t)=y(t)\left[\frac{m \beta x(t)}{\alpha+x(t)}-d\right]-E(t) y(t),
\end{aligned}
$$

where $x(t)$ and $y(t)$ represent population density of prey and predator population with time $t$, respectively. $r$ is the intrinsic growth rate of prey, $K$ stands for maximum environmental capacity, $\beta$ denotes predation rate of predator, $m$ represents biomass transmission rate from predation activity, and $d$ is death rate of predator.

It is well known that commercial harvesting fluctuates with dynamical variation of economic interest [14, 15]. Although the harvested biological models have received great attention from both mathematical and theoretical biologists, little work has been done on dynamic effect of economic interest on harvested prey-predator fishery model [14, 15]. In 1954, Gordon proposes common-property resource economic theory [14], which investigates dynamic effect of commercial harvesting on ecosystem from an economic perspective $[14,15]$. In [14], an algebraic equation is proposed to investigate economic interest of commercial harvesting:

$$
\begin{aligned}
& \text { Net Economic Revenue } \\
& =\text { Total Revenue (TR) - Total Cost (TC) } .
\end{aligned}
$$

In order to show dynamical variation of unit price of harvested population due to change of amount of harvested population, $a_{1} /\left(b_{1}+E(t) x(t)\right)$ is assumed to be unit price of commercially harvested prey, and $a_{2} /\left(b_{2}+E(t) y(t)\right)$ is assumed to be unit price of commercially harvested predator. Hence, TR in (2) takes the following form TR = $a_{1} E(t) x(t) /\left(b_{1}+E(t) x(t)\right)+a_{2} E(t) y(t) /\left(b_{2}+E(t) y(t)\right)$, where $a_{1}, a_{2}, b_{1}, b_{2}$ are all positive constants. Let $c$ represent cost of 
commercial harvesting. Hence, TC in (2) takes the following form $\mathrm{TC}=c E(t)$.

Recently, many theoreticians and experimentalists have investigated complex dynamics of prey-predator fishery system $[16,17]$; it reveals that time delay may cause the loss of stability and other complicated dynamical behavior such as the periodic structure and bifurcation phenomenon $[15,18$, 19].

Keeping these aspects in view, we will extend work in [5] by incorporating maturation delay for prey and gestation delay for predator into system (1). In this paper, prey is considered to be delayed by maturation delay $\tau_{1} \geq 0$ due to population crowding $[15,16]$, and predator is assumed to be delayed by gestation delay $\tau_{2} \geq 0$ [16]. Based on (1) and (2), a singular prey-predator fishery model is established as follows:

$$
\begin{aligned}
& \dot{x}(t)=r x(t)\left(1-\frac{x\left(t-\tau_{1}\right)}{K}\right)-\frac{\beta x(t) y(t)}{\alpha+x(t)}-E(t) \\
& \cdot x(t), \\
& \dot{y}(t)=y(t)\left[\frac{m \beta x\left(t-\tau_{2}\right)}{\alpha+x\left(t-\tau_{2}\right)}-d\right]-E(t) y(t), \\
& 0=E(t) \\
& \cdot\left[x(t) \frac{a_{1}}{b_{1}+E(t) x(t)}+y(t) \frac{a_{2}}{b_{2}+E(t) y(t)}-c\right] \\
& -v,
\end{aligned}
$$

where $c$ represents cost of commercial harvesting, $v$ is assumed to be economic interest of commercial harvesting, $E(t)$ represents commercial harvesting amount with respect to time $t$, and interpretations for other parameters and state variables share the same interpretations introduced in system (1) and (2). Furthermore, system (3) is investigated with initial conditions

$$
\begin{aligned}
x(\theta) & \geq 0, \\
y(0) & \geq 0, \\
E(0) & \geq 0, \\
\theta & \in[-\tau, 0], \tau=\max \left\{\tau_{1}, \tau_{2}\right\} .
\end{aligned}
$$

System (3) is rewritten as follows:

$$
\begin{aligned}
& \Xi\left[\begin{array}{c}
\dot{x}(t) \\
\dot{y}(t) \\
0
\end{array}\right] \\
& =\left[\begin{array}{c}
r x(t)\left(1-\frac{x\left(t-\tau_{1}\right)}{K}\right)-\frac{\beta x(t) y(t)}{\alpha+x(t)}-E(t) x(t) \\
y(t)\left[\frac{m \beta x\left(t-\tau_{2}\right)}{\alpha+x\left(t-\tau_{2}\right)}-d\right]-E(t) y(t) \\
E(t)\left[x(t) \frac{a_{1}}{b_{1}+E(t) x(t)}+y(t) \frac{a_{2}}{b_{2}+E(t) y(t)}-c\right]-v
\end{array}\right],
\end{aligned}
$$

where the third equation in system (3) does not contain any differentiated variables; hence the third row in leading matrix $\Xi=\left[\begin{array}{lll}1 & 0 & 0 \\ 0 & 1 & 0 \\ 0 & 0 & 0\end{array}\right]$ has a zero row.
The rest of the sections of this paper are organized as follows. Positivity of any solution of system (3) and uniform persistence of system (3) are investigated in the second section. Local stability analysis of system (3) around interior equilibrium is discussed in the third section. The optimal system is derived and solved numerically based on an iterative method with Runge-Kutta fourth order scheme. Optimal harvest control problems associated with maximizing total discounted net revenues from the fishery and minimizing harvest cost are discussed in the fourth section. Numerical simulations are carried out to support theoretical analysis in the fifth section. Finally, this paper ends with a conclusion.

\section{Positivity and Uniform Persistence}

Theorem 1. Any solutions of system (3) with initial conditions (4) are positive.

Proof. Due to lemma in [20] and Theorem A.4 in [21], any solution of system (3) with initial conditions (4) exists uniquely and each component of solution remains within interval $\left[0, X_{0}\right)$ for some $X_{0}>0$. Standard and simple arguments show that any solution of system (3) always exists and stays positive.

Theorem 2. If $v>0, \tau_{1}$ and $\tau_{2}$ are bounded, and the following inequalities (6) hold, and then system (3) with initial conditions (4) is uniformly persistent:

$$
\begin{aligned}
& m \beta K e^{r \tau_{1}}>\alpha d, \\
& a_{1} b_{2} \beta K e^{r \tau_{1}}+a_{2} b_{1} r\left(\alpha+K e^{r \tau_{1}}\right)>c b_{1} b_{2} \beta, \\
& c \alpha b_{1}>a_{1} K e^{r \tau_{1}}\left(m \beta K e^{r \tau_{1}}-\alpha d\right), \\
& c b_{2} \alpha \beta>a_{2} r\left(\alpha+K e^{r \tau_{1}}\right)\left(m \beta K e^{r \tau_{1}}-\alpha d\right) .
\end{aligned}
$$

Proof. By using Taylor series expansion [2], for $x(t)$ and $\tau_{1}>$ $0, \tau_{2}>0$ we have

$$
\begin{aligned}
& x\left(t-\tau_{1}\right)=x(t)-\tau_{1} \frac{d}{d t}\left(x(t)-\tau_{1} \dot{x}(t)+\cdots\right), \\
& x\left(t-\tau_{2}\right)=x(t)-\tau_{2} \frac{d}{d t}\left(x(t)-\tau_{2} \dot{x}(t)+\cdots\right) .
\end{aligned}
$$

Consequently, it can be derived that

$$
\begin{aligned}
& x\left(t-\tau_{1}\right) \leq x(t), \\
& x\left(t-\tau_{2}\right) \leq x(t) .
\end{aligned}
$$
that

By using (8) and the first equation of system (3), it gives

$$
\begin{aligned}
& x(t) \leq x\left(t-\tau_{1}\right) e^{r \tau_{1}}, \\
& x(t) \leq r x(t)\left(1-\frac{x(t)}{K e^{r \tau_{1}}}\right),
\end{aligned}
$$

which derives that

$$
\limsup _{t \rightarrow \infty} x(t) \leq K e^{r \tau_{1}}:=\mathrm{Q}_{1} .
$$


If $\tau_{1}$ is bounded, then it is easy to show that $Q_{1}>0$ and is bounded. According to (10), it can be derived that there exists $T_{1}>0$ such that $x(t) \leq Q_{1}$ holds for $t>T_{1}+\tau_{1}$.

Based on the first equation of system (3) and practical interpretations of prey survival, it can be derived that there exists $T_{2}>T_{1}+\tau_{1}$ such that $y(t) \leq r[\alpha+x(t)] / \beta$, and

$$
\limsup _{t \rightarrow \infty} y(t) \leq \frac{r[\alpha+x(t)]}{\beta} \leq \frac{r\left(\alpha+K e^{r \tau_{1}}\right)}{\beta}:=Q_{2} \text {. }
$$

Similarly, based on the second equation of system (3) and practical interpretations of predator survival, it is shown that there exists $T_{3}>T_{2}$ such that

$$
E(t) \leq \frac{m \beta x\left(t-\tau_{2}\right)}{\alpha}-d
$$

By using (8), if $\tau_{2}$ is bounded then it follows that $E(t) \leq$ $(m \beta x(t) / \alpha)-d$, and

$$
\limsup _{t \rightarrow \infty} E(t) \leq \frac{m \beta K e^{r \tau_{1}}-\alpha d}{\alpha}:=Q_{3} .
$$

If $m \beta K e^{r \tau_{1}}>\alpha d$, then it can be derived that $Q_{3}>0$ and is bounded. According to (13), it is shown that there exists $T_{4}>T_{3}$ such that $E(t) \leq Q_{3}$ holds for $t>T_{4}$.

By virtue of the third equation of system (3), when $v>0$, it can be derived that there exists $T_{5}>T_{4}$ such that

$$
\begin{aligned}
\frac{v}{E(t)}= & x(t) \frac{a_{1}}{b_{1}+E(t) x(t)}+y(t) \frac{a_{2}}{b_{2}+E(t) y(t)} \\
& -c \leq \frac{a_{1} x(t)}{b_{1}}+\frac{a_{2} y(t)}{b_{2}}-c,
\end{aligned}
$$

which implies that

$$
\begin{aligned}
& \liminf _{t \rightarrow \infty} E(t) \\
& \geq \frac{v b_{1} b_{2} \beta}{a_{1} b_{2} \beta K e^{r \tau_{1}}+a_{2} b_{1} r\left(\alpha+K e^{r \tau_{1}}\right)-c b_{1} b_{2} \beta}:=W_{3} .
\end{aligned}
$$

If $a_{1} b_{2} \beta K e^{r \tau_{1}}+a_{2} b_{1} r\left(\alpha+K e^{r \tau_{1}}\right)>c b_{1} b_{2} \beta$, then it is easy to show that $W_{3}>0$ and is bounded, and then there exists $T_{6}>T_{5}$ such that $E(t) \geq W_{3}$ holds for $t>T_{6}$.

By virtue of the third equation of system (3), when $v>0$, it can be derived that there exists $T_{7}>T_{6}$ such that

$$
\begin{aligned}
& E(t) x(t)>\frac{b_{1}}{a_{1}}\left[c-\frac{a_{2}}{b_{2}} E(t) y(t)\right], \\
& E(t) y(t)>\frac{b_{2}}{a_{2}}\left[c-\frac{a_{1}}{b_{1}} E(t) x(t)\right] .
\end{aligned}
$$

According to (10), (11), and (13), it can be obtained that

$$
\begin{aligned}
& \liminf _{t \rightarrow \infty} x(t) \\
& \geq \frac{c b_{2} \alpha \beta-a_{2} r\left(\alpha+K e^{r \tau_{1}}\right)\left(m \beta K e^{r \tau_{1}}-\alpha d\right)}{\alpha \beta}:=W_{1}, \\
& \liminf _{t \rightarrow \infty} y(t) \geq \frac{b_{2}\left[\alpha c b_{1}-a_{1} K e^{r \tau_{1}}\left(m \beta K e^{r \tau_{1}}-\alpha d\right)\right]}{a_{2} b_{1}\left(m \beta K e^{r \tau_{1}}-\alpha d\right)} \\
& \quad:=W_{2} .
\end{aligned}
$$

If the following two inequalities hold

$$
\begin{aligned}
c \alpha b_{1} & >a_{1} K e^{r \tau_{1}}\left(m \beta K e^{r \tau_{1}}-\alpha d\right), \\
c b_{2} \alpha \beta & >a_{2} r\left(\alpha+K e^{r \tau_{1}}\right)\left(m \beta K e^{r \tau_{1}}-\alpha d\right),
\end{aligned}
$$

then $W_{1}>0$ and $W_{2}>0$ and is bounded, and there exists $T_{8}>T_{7}$ such that $x(t) \geq W_{1}$ and $y(t) \geq W_{2}$ hold for $t>T_{8}$.

Based on the above analysis, it can be concluded that system (3) with initial conditions (4) is uniformly persistent.

\section{Local Stability Analysis}

Firstly, interior equilibrium $P^{*}$ of system (3) in the case of $v>$ 0 is computed as follows: $P^{*}\left(x^{*}, y^{*}, E^{*}\right)=\left(x^{*},\left(\alpha+x^{*}\right)[K d+\right.$ $\left.\left.r\left(K-x^{*}\right)\right] / K \beta-m x^{*}, m \beta x^{*} /\left(\alpha+x^{*}\right)-d\right)$, and $x^{*}$ satisfy the following equation:

$$
\begin{aligned}
& A_{0} x^{* 6}+A_{1} x^{* 5}+A_{2} x^{* 4}+A_{3} x^{* 3}+A_{4} x^{* 2}+A_{5} x^{*} \\
& +A_{6}=0,
\end{aligned}
$$

where $A_{k}(k=0,1, \ldots, 6)$ are defined as follows:

$$
\begin{aligned}
& A_{0}=r(d-m \beta)^{2}\left(a_{1}+a_{2}-v+d-m \beta\right), \\
& A_{1}=(m \beta-d)^{2}[3 \alpha d r \\
& +(K d+K r-\alpha r-m K \beta)\left(m \beta-d+v-a_{1}-a_{2}\right) \\
& \left.-c b_{1} r\right] \\
& A_{2}=K \alpha(d+r)(m \beta-d)^{3}-3 \alpha d(m \beta-d)^{2}(K d \\
& +K r-\alpha r-m K \beta)-3 \alpha^{2} d^{2} r(m \beta-d)+(m \beta \\
& -d)^{2}\left[c b_{1}(K d+K r-\alpha r-m K \beta)+K \beta c b_{2}\right. \\
& \left.-\alpha K(d+r)\left(a_{1}+a_{2}-v\right)\right]+\alpha(m \beta-d)(m \beta \\
& -3 d)\left[\left(a_{1}+a_{2}-v\right)(K d+K r-\alpha r-m K \beta)\right. \\
& \left.-c b_{1} r\right] \\
& A_{3}=\alpha K(d+r)\left(c b_{1}-3 \alpha d\right)(m \beta-d)^{2}+r \alpha^{3} d^{3} \\
& +K c b_{1} b_{2} \beta(m \beta-d)+v b_{1} b_{2} K \beta+\alpha(m \beta-d)(K d \\
& +K r-\alpha r-m K \beta)\left[3 \alpha d^{2}+c b_{1}(m \beta-3 d)\right]+(v \\
& \left.-a_{1}\right) b_{2} K \beta(m \beta-d)+r d \alpha^{2}(3 d-2 m \beta) \\
& \cdot\left[\left(a_{1}+a_{2}-v\right)(K d+K r-\alpha r-m K \beta)-c b_{1} r\right] \\
& +\alpha(m \beta-d)(m \beta-3 d)\left[K \beta c b_{2}\right. \\
& \left.-\alpha K(d+r)\left(a_{1}+a_{2}-v\right)\right] \text {, }
\end{aligned}
$$




$$
\begin{aligned}
A_{4} & =\alpha^{3} d^{3}[3 K(d+r)(m \beta-d) \\
& -d(K d+K r-\alpha r-m K \beta)]+b_{2} K \beta \alpha^{2}(m \beta-d) \\
& \cdot\left(v-a_{1}\right)+c b_{1} \alpha k(d+r)(m \beta-d)(m \beta-3 d) \\
& \cdot\left[K \beta c b_{2}+c b_{1}(K r+K d-a \alpha r-m K \beta)\right]+2\left(a_{1}\right. \\
& -v) b_{2} K \alpha \beta(m \beta-d)+2\left(a_{2}-v\right) b_{1} d \alpha^{2} K(d+r \\
& -m \beta)+b_{2} K \alpha \beta\left(v-a_{1}\right)(\alpha-2)(m \beta-d), \\
A_{5} & =c b_{1} \alpha^{3} d K(d+r)(3 d-2 m \beta)+b_{2} K d \alpha^{3} \beta\left(a_{1}\right. \\
& -v)+\alpha^{3} d^{3}\left[K \beta c b_{2}-\alpha K(d+r)\left(a_{1}+a_{2}-v\right)\right. \\
& \left.+c b_{1}(K r+K d-\alpha r-m K \beta)\right]+b_{1}\left(v-a_{2}\right) \\
& \cdot\left[\alpha^{3} K(d+r)(m \beta-d)-m \beta K-2 \alpha^{2} d K(d+r)\right], \\
A_{6} & =c b_{1} \alpha^{4} K d^{2}(d+r)+\left(a_{2}-v\right) b_{1} \alpha^{3} K(d+r) \\
& +K \beta b_{1} b_{2} \alpha^{3}(v-c d) .
\end{aligned}
$$

Furthermore, it follows from the practical interpretations of interior equilibrium that $P^{*}$ exists provided that $y^{*}>0$ and $E^{*}>0$, which derives that

$$
\begin{array}{r}
r x^{* 2}+(\alpha r+K m \beta-K d-K r) x^{*}-\alpha K(d+r)<0, \\
(m \beta-d) x^{*}-\alpha d>0 .
\end{array}
$$

It follows from Routh-Hurwitz criterion [21], simple sufficient conditions for existence of $P^{*}$ of system (3) are as follows:

$$
\begin{aligned}
0< & v<\min \left\{a_{1}+a_{2}+d\right. \\
& \left.-m \beta, \frac{\left(1-a_{2}\right) b_{2} \beta-d \alpha(d+r)}{b_{2} \beta-d-r}\right\} .
\end{aligned}
$$

Based on Jacobian matrix of system (3) evaluated around $P^{*}$ and $\Xi$ defined in (5), characteristic equation of system (3) around $P^{*}$ is as follows:

$$
\lambda^{2}+B_{1} \lambda+B_{2}+\left(B_{3} \lambda+B_{4}\right) e^{-\lambda \tau_{1}}+B_{5} \mathrm{e}^{-\lambda \tau_{2}}=0,
$$

where $B_{i}, i=1,2, \ldots, 5$, are defined as follows:

$$
\begin{aligned}
& B_{1}=-\frac{\beta x^{*} y^{*}}{\left(\alpha+x^{*}\right)^{2}}-\frac{a_{2} b_{2} E^{*} y^{*}\left(b_{1}+E^{*} x^{*}\right)^{2}+a_{1} b_{1} E^{*} x^{*}\left(b_{2}+E^{*} y^{*}\right)^{2}}{a_{1} b_{1} x^{*}\left(b_{2}+E^{*} y^{*}\right)^{2}+a_{2} b_{2} y^{*}\left(b_{1}+E^{*} x^{*}\right)^{2}-c\left(b_{1}+E^{*} x^{*}\right)^{2}\left(b_{2}+E^{*} y^{*}\right)^{2}}, \\
& B_{2}=\frac{a_{2} b_{2} \beta x^{*} y^{* 2} E^{*}\left(b_{1}+E^{*} x^{*}\right)^{2}+a_{1} b_{1} \beta x^{*} y^{*} E^{*}\left(\alpha+x^{*}\right)\left(b_{2}+E^{*} y^{*}\right)^{2}}{\left(\alpha+x^{*}\right)^{2}\left[a_{1} b_{1} x^{*}\left(b_{2}+E^{*} y^{*}\right)^{2}+a_{2} b_{2} y^{*}\left(b_{1}+E^{*} x^{*}\right)^{2}-c\left(b_{1}+E^{*} x^{*}\right)^{2}\left(b_{2}+E^{*} y^{*}\right)^{2}\right]}, \\
& B_{3}=\frac{r x^{*}}{K}, \\
& B_{4}=-\frac{r a_{2} b_{2} x^{*} y^{*} E^{*}\left(b_{1}+E^{*} x^{*}\right)^{2}}{K\left[a_{1} b_{1} x^{*}\left(b_{2}+E^{*} y^{*}\right)^{2}+a_{2} b_{2} y^{*}\left(b_{1}+E^{*} x^{*}\right)^{2}-c\left(b_{1}+E^{*} x^{*}\right)^{2}\left(b_{2}+E^{*} y^{*}\right)^{2}\right]}, \\
& B_{5}=\frac{m \alpha \beta x^{*} y^{*}}{\left(\alpha+x^{*}\right)^{2}}\left[\frac{\beta}{\alpha+x^{*}}-\frac{a_{2} b_{2} E^{*}\left(b_{1}+E^{*} x^{*}\right)}{a_{1} b_{1} x^{*}\left(b_{2}+E^{*} y^{*}\right)^{2}+a_{2} b_{2} y^{*}\left(b_{1}+E^{*} x^{*}\right)^{2}-c\left(b_{1}+E^{*} x^{*}\right)^{2}\left(b_{2}+E^{*} y^{*}\right)^{2}}\right] .
\end{aligned}
$$

3.1. Case I: $\tau_{1}>0, \tau_{2}=0$. When $\tau_{1}>0$ and $\tau_{2}=0$, (23) can be rewritten as follows:

$$
\lambda^{2}+B_{1} \lambda+B_{2}+B_{5}+\left(B_{3} \lambda+B_{4}\right) e^{-\lambda \tau_{1}}=0 .
$$

By substituting $\lambda=i \omega_{1}\left(\omega_{1}>0\right)$ into (25) and separating real and imaginary parts, it gives that

$$
\begin{aligned}
\omega_{1}^{2}-\left(B_{2}+B_{5}\right) & =B_{4} \cos \left(\omega_{1} \tau_{1}\right)+B_{3} \omega_{1} \sin \left(\omega_{1} \tau_{1}\right), \\
B_{1} \omega_{1} & =-B_{3} \omega_{1} \cos \left(\omega_{1} \tau_{1}\right)+B_{4} \sin \left(\omega_{1} \tau_{1}\right),
\end{aligned}
$$

which derives that

$$
\begin{aligned}
\omega_{1}^{4} & +\left[B_{1}^{2}-2\left(B_{2}+B_{5}\right)-B_{3}^{2}\right] \omega_{1}^{2}+\left(B_{2}+B_{5}\right)^{2}-B_{4}^{2} \\
& =0 .
\end{aligned}
$$

According to Routh-Hurwitz criterion [21], a simple sufficient condition $\left(B_{2}+B_{5}\right)^{2}-B_{4}^{2}<0$ guarantees existence of positive root $\omega_{1}^{*}$ of (27). Hence, (25) has a pair of purely imaginary roots of the form $\pm i \omega_{1}^{*}$.

By eliminating $\sin \left(\omega_{1} \tau_{1}\right)$ from (26), it can be calculated that $\tau_{1 k}^{*}$ corresponding to $\omega_{1}^{*}$ is as follows:

$$
\begin{aligned}
\tau_{1 k}^{*}= & \frac{1}{\omega_{1}^{*}} \arccos \left\{\frac{\left(B_{4}-B_{1} B_{3}\right) \omega_{1}^{* 2}-\left(B_{2}+B_{5}\right) B_{4}}{B_{4}^{2}+B_{3}^{2} \omega_{1}^{* 2}}\right\} \\
& +\frac{2 k \pi}{\omega_{1}^{*}},
\end{aligned}
$$

where $k=0,1, \ldots$ 
It follows from Butler's lemma [22] that system (3) is locally stable around $P^{*}$ when $0<\tau_{1}<\tau_{10}^{*}, \tau_{2}=0$.

Based on the above analysis, local stability analysis of system (3) around $P^{*}$ is concluded in Theorem 3.

Theorem 3. If $\left(B_{2}+B_{5}\right)^{2}-B_{4}^{2}<0$, then system (3) is locally stable around $P^{*}$ when $0<\tau_{1}<\tau_{10}^{*}, \tau_{2}=0$, and $\tau_{10}^{*}$ is defined in (28).

3.2. Case II: $\tau_{1}=0, \tau_{2}>0$. When $\tau_{1}=0, \tau_{2}>0$, by using similar arguments in Section 3.1, local stability analysis of system (3) around $P^{*}$ can be concluded in the following Theorem 4.

Theorem 4. If $\left(B_{2}+B_{4}\right)^{2}-B_{5}^{2}<0$, then system (3) is locally stable around $P^{*}$ when $\tau_{1}=0,0<\tau_{2}<\tau_{20}^{*}$, and $\tau_{20}^{*}$ is defined as follows:

$$
\tau_{2 k}^{*}=\frac{1}{\omega_{2}^{*}} \arccos \left\{\frac{\omega_{2}^{* 2}-B_{2}-B_{4}}{B_{5}}\right\}+\frac{2 k \pi}{\omega_{2}^{*}},
$$

where $k=0,1, \ldots$ and $\omega_{2}^{*}$ is a positive root of the following equation:

$$
\begin{aligned}
\omega_{2}^{4} & +\left[\left(B_{1}+B_{3}\right)^{2}-2\left(B_{2}+B_{4}\right)\right] \omega_{2}^{2}+\left(B_{2}+B_{4}\right)^{2}-B_{5}^{2} \\
& =0
\end{aligned}
$$

3.3. Case III: $\tau_{1}>0, \tau_{2} \in\left(0, \tau_{20}^{*}\right), \tau_{1} \neq \tau_{2}$. In this subsection, $\tau_{1}$ is considered to be bifurcation parameter and $\tau_{2}$ is regarded as fixed value $\tilde{\tau}_{2} \in\left(0, \tau_{20}^{*}\right)$, where $\tau_{20}^{*}$ is determined in Theorem 4 .

Let $\lambda=i \sigma_{1}\left(\sigma_{1}>0\right)$ be the root of (23), and two transcendental equations are obtained as follows:

$$
\begin{aligned}
\sigma_{1}^{2} & -\left(B_{2}+B_{5} \cos \left(\sigma_{1} \widetilde{\tau}_{2}\right)\right) \\
& =B_{4} \cos \left(\sigma_{1} \tau_{1}\right)+B_{3} \sigma_{1} \sin \left(\sigma_{1} \tau_{1}\right), \\
B_{1} & \sigma_{1}-B_{5} \sin \left(\sigma_{1} \widetilde{\tau}_{2}\right) \\
& =B_{4} \sin \left(\sigma_{1} \tau_{1}\right)-B_{3} \sigma_{1} \cos \left(\sigma_{1} \tau_{1}\right),
\end{aligned}
$$

where $B_{k}, k=1,2, \ldots, 5$, are defined in (23).

By eliminating $\widetilde{\tau}_{2}$ from (31), it can be derived that

$$
\begin{aligned}
& \cos \left(\sigma_{1} \tau_{1}\right)=\frac{f_{10}\left(\sigma_{1}, \tilde{\tau}_{2}\right)}{f_{12}\left(\sigma_{1}, \tilde{\tau}_{2}\right)}, \\
& \sin \left(\sigma_{1} \tau_{1}\right)=\frac{f_{11}\left(\sigma_{1}, \tilde{\tau}_{2}\right)}{f_{12}\left(\sigma_{1}, \tilde{\tau}_{2}\right)},
\end{aligned}
$$

where $f_{1 k}\left(\sigma_{1}, \tilde{\tau}_{2}\right), k=0,1,2$, are defined as follows:

$$
\begin{aligned}
f_{10}\left(\sigma_{1}, \tilde{\tau}_{2}\right)= & \left(B_{4}-B_{1} B_{3}\right) \sigma_{1}^{2}-B_{2} B_{4} \\
& -B_{4} B_{5} \cos \left(\sigma_{1} \widetilde{\tau}_{2}\right) \\
& +B_{3} B_{5} \sigma_{1} \sin \left(\sigma_{1} \tilde{\tau}_{2}\right), \\
f_{11}\left(\sigma_{1}, \widetilde{\tau}_{2}\right)= & B_{3} \sigma_{1}^{3}+\left(B_{1} B_{4}-B_{2} B_{3}\right) \sigma_{1}
\end{aligned}
$$

$$
\begin{aligned}
& -B_{3} B_{5} \sigma_{1} \cos \left(\sigma_{1} \widetilde{\tau}_{2}\right) \\
& -B_{4} B_{5} \sin \left(\sigma_{1} \widetilde{\tau}_{2}\right), \\
f_{12}\left(\sigma_{1}, \widetilde{\tau}_{2}\right)= & B_{4}^{2}+B_{3}^{2} \sigma_{1}^{2} .
\end{aligned}
$$

Based on $f_{1 k}\left(\sigma_{1}, \tilde{\tau}_{2}\right), k=0,1,2$, defined in (32), we define

$$
\begin{aligned}
F_{1}\left(\sigma_{1}, \tilde{\tau}_{2}\right) & =f_{10}^{2}\left(\sigma_{1}, \tilde{\tau}_{2}\right)+f_{11}^{2}\left(\sigma_{1}, \widetilde{\tau}_{2}\right)-f_{12}^{2}\left(\sigma_{1}, \widetilde{\tau}_{2}\right) \\
& =0
\end{aligned}
$$

It is difficult to discuss root properties of transcendental equation (34) due to its complicated form. Without investigating the properties of roots of (34) in detail, it follows from simple computations that (23) has a pair of purely imaginary roots when (34) has at least two roots $0<\sigma_{10}<\sigma_{11}<\cdots$.

By denoting $\sigma_{1 w}=\max \left\{\sigma_{1 k}, k=0,1, \ldots\right\}$ and $\tau_{1}$ which is considered to be bifurcation parameter, we can obtain the corresponding critical value $\tau_{1 w}$ :

$$
\begin{aligned}
\tau_{1 k} & =\frac{\psi_{1 w}+2 k \pi}{\sigma_{1 w}}, \\
\tau_{1 w} & =\min \left\{\tau_{1 k}, k=0,1, \ldots\right\},
\end{aligned}
$$

where $k=0,1, \ldots, \psi_{1 c} \in[0,2 \pi)$ satisfies the following equations:

$$
\begin{aligned}
& \cos \psi_{1 w}=\frac{f_{10}\left(\sigma_{1 w}, \tilde{\tau}_{2}\right)}{f_{12}\left(\sigma_{1 w}, \tilde{\tau}_{2}\right)}, \\
& \sin \psi_{1 w}=\frac{f_{11}\left(\sigma_{1 w}, \tilde{\tau}_{2}\right)}{f_{12}\left(\sigma_{1 w}, \tilde{\tau}_{2}\right)} .
\end{aligned}
$$

Based on the above analysis, local stability analysis around $P^{*}$ due to dynamical variation of bifurcation parameter $\tau_{1}$ can be summarized as follows.

Theorem 5. If (34) has at least two roots $0<\sigma_{10}<\sigma_{11}<\cdots$, and there exists $\tau_{1 w}$ defined in (35), then system (3) is locally stable around $P^{*}$ when $0<\tau_{1}<\tau_{1 w}, \tau_{2}=\widetilde{\tau}_{2}$.

3.4. Case IV: $\tau_{2}>0, \tau_{1} \in\left(0, \tau_{10}^{*}\right), \tau_{1} \neq \tau_{2}$. In this subsection, $\tau_{2}$ is considered to bifurcation parameter and $\tau_{1}$ is regarded as fixed value $\widetilde{\tau}_{1} \in\left(0, \tau_{10}^{*}\right)$, where $\tau_{10}^{*}$ is determined in Theorem 3. By using similar arguments in Theorem 5 of this paper, local stability analysis around $P^{*}$ can be concluded in Theorem 6.

Firstly, we define following equation:

$$
\begin{aligned}
F_{2}\left(\sigma_{2}, \tilde{\tau}_{1}\right) & =f_{20}^{2}\left(\sigma_{2}, \tilde{\tau}_{1}\right)+f_{21}^{2}\left(\sigma_{2}, \tilde{\tau}_{1}\right)-f_{22}^{2}\left(\sigma_{2}, \widetilde{\tau}_{1}\right) \\
& =0
\end{aligned}
$$

where $f_{2 k}\left(\sigma_{2}, \tilde{\tau}_{2}\right), k=0,1,2$, are defined as follows:

$$
\begin{aligned}
f_{20}\left(\sigma_{2}, \tilde{\tau}_{1}\right)= & \sigma_{2}^{2}-B_{2}-B_{4} \cos \left(\sigma_{2} \tilde{\tau}_{1}\right) \\
& -B_{3} \sigma_{2} \sin \left(\sigma_{2} \widetilde{\tau}_{1}\right),
\end{aligned}
$$




$$
\begin{aligned}
f_{21}\left(\sigma_{2}, \widetilde{\tau}_{1}\right)= & B_{1} \sigma_{2}+B_{3} \sigma_{2} \cos \left(\sigma_{2} \widetilde{\tau}_{1}\right) \\
& -B_{4} \sin \left(\sigma_{2} \widetilde{\tau}_{1}\right), \\
f_{22}\left(\sigma_{2}, \widetilde{\tau}_{1}\right)= & B_{5} .
\end{aligned}
$$

Theorem 6. If (37) has at least two roots $0<\sigma_{20}<\sigma_{21}<$ $\cdots$, there exists a critical delay $\tau_{2 w}$, and then system (3) is locally stable around $P^{*}$ when $0<\tau_{2}<\tau_{2 w}, \tau_{1}=\tilde{\tau}_{1}$, where $\tau_{2 w}=\min \left\{\left(\psi_{2 w}+2 k \pi\right) / \sigma_{2 w}, k=0,1, \ldots\right\}, \sigma_{2 w}=\max \left\{\sigma_{2 k}, k=\right.$ $0,1, \ldots\}$ and $\psi_{2 w} \in[0,2 \pi)$ satisfies the following equations:

$$
\begin{aligned}
& \cos \psi_{2 w}=\frac{f_{20}\left(\sigma_{2 w}, \widetilde{\tau}_{1}\right)}{f_{22}\left(\sigma_{2 w}, \widetilde{\tau}_{1}\right)}, \\
& \sin \psi_{2 w}=\frac{f_{21}\left(\sigma_{2 w}, \widetilde{\tau}_{1}\right)}{f_{22}\left(\sigma_{2 w}, \widetilde{\tau}_{1}\right)} .
\end{aligned}
$$

\section{Optimal Control Problem}

In this section, $\tau_{1}$ and $\tau_{2}$ are considered to be a fixed value $\widehat{\tau}_{1} \in\left(0, \tau_{1 w}\right)$ and $\widehat{\tau}_{2} \in\left(0, \tau_{20}^{*}\right)$, respectively; $\widehat{\tau}_{1} \neq \widehat{\tau}_{2}, \tau_{1 w}$, and $\tau_{20}^{*}$ have been determined in Theorems 5 and 4, respectively. It follows from Theorem 5 that system (3) with $\widehat{\tau}_{1}$ and $\widehat{\tau}_{2}$ is locally stable around $P^{*}$, and we will investigate optimal control $E(t)$ with maximizing total discounted net revenues and minimizing commercial harvesting cost during $\left[t_{0}, t_{f}\right]$. The optimal harvest control problem is formulated as follows:

$$
\begin{aligned}
& J(E)=\int_{t_{0}}^{t_{f}} e^{-\delta t}\left[E(t) x(t) \frac{a_{1}}{b_{1}+E(t) x(t)}\right. \\
& \left.+E(t) y(t) \frac{a_{2}}{b_{2}+E(t) y(t)}-c E(t)\right] d t
\end{aligned}
$$

where $\delta$ is instantaneous annual discount rate [7], and optimal control problem (40) is subject to the first and second delayed differential equations of system (3) with $\widehat{\tau}_{1}$ and $\widehat{\tau}_{2}$. Furthermore, convexity of $J(E)$ with respect to $E(t)$, linearity of the delayed differential equations with $E(t)$, and range values compactness of state variables can be uniformly investigated when discussing existence of optimal harvest control $E(t)$. By assuming $E_{\delta}$ as an optimal harvest control with corresponding optimal state variables $x_{\delta}$ and $y_{\delta}$ of system (3) with $\widehat{\tau}_{1}$ and $\widehat{\tau}_{2}$, and $E_{\delta}$ satisfies the following condition:

$$
J\left(E_{\delta}\right)=\max \{J(E(t)) \mid E(t) \in U\}
$$

where $U$ represents control set that is defined as follows:

$$
\begin{aligned}
U= & \left\{E:\left[t_{0}, t_{f}\right]\right. \\
& \left.\longrightarrow\left[0, E_{\max }\right] \mid E \text { is Lebesgue measurable. }\right\}
\end{aligned}
$$

The Hamiltonian function $H(t)$ associated with system (3) with $\widehat{\tau}_{1}$ and $\widehat{\tau}_{2}$ is

$$
\begin{aligned}
& H(t)=e^{-\delta t}\left[E(t) x(t) \frac{a_{1}}{b_{1}+E(t) x(t)}\right. \\
& \left.+E(t) y(t) \frac{a_{2}}{b_{2}+E(t) y(t)}-c E(t)\right]+\lambda_{1}(t) \\
& \quad .\left[r x(t)\left(1-\frac{x_{\widehat{\tau}_{1}}}{K}\right)-\frac{\beta x(t) y(t)}{\alpha+x(t)}-E(t) x(t)\right] \\
& +\lambda_{2}(t)\left[y(t)\left(\frac{m \beta x_{\widehat{\tau}_{2}}}{\alpha+x_{\widehat{\tau}_{2}}}-d\right)-E(t) y(t)\right],
\end{aligned}
$$

where $x_{\widehat{\tau}_{1}}:=x\left(t-\widehat{\tau}_{1}\right), x_{\widehat{\tau}_{2}}:=x\left(t-\widehat{\tau}_{2}\right)$, and $\lambda_{1}(t), \lambda_{2}(t)$ are adjoint functions.

Theorem 7. There exists an optimal harvest $E_{\delta}$ which is determined by (44); furthermore, there exist adjoint functions $\lambda_{1}(t)$, $\lambda_{2}(t)$ satisfying system (47) with $\lambda_{k}\left(t_{f}\right)=0, k=1,2$.

$$
\begin{aligned}
& \frac{a_{1} b_{1} x_{\delta}}{\left(b_{1}+E_{\delta} x_{\delta}\right)^{2}}+\frac{a_{2} b_{2} y_{\delta}}{\left(b_{2}+E_{\delta} y_{\delta}\right)^{2}} \\
& =c+\left[x_{\delta} \lambda_{1}(t)+y_{\delta} \lambda_{2}(t)\right] e^{\delta t}
\end{aligned}
$$

Proof. By differentiating $H(t)$, the adjoint system can be obtained as follows:

$$
\begin{aligned}
\frac{d \lambda_{1}}{d t}= & -\frac{\partial H}{\partial x}(t)-\chi_{1\left[t_{0}, t_{f}-\widehat{\tau}_{1}\right]}(t) \frac{\partial H}{\partial x_{\widehat{\tau}_{1}}}\left(t+\widehat{\tau}_{1}\right) \\
& -\chi_{2\left[t_{0}, t_{f}-\widehat{\tau}_{2}\right]}(t) \frac{\partial H}{\partial x_{\widehat{\tau}_{2}}}\left(t+\widehat{\tau}_{2}\right), \\
\frac{d \lambda_{2}}{d t}= & -\frac{\partial H}{\partial y}(t),
\end{aligned}
$$

where

$$
\begin{aligned}
& \chi_{1\left[t_{0}, t_{f}-\widehat{\tau}_{1}\right]}(t)= \begin{cases}1, & t \in\left[0, t_{f}-\widehat{\tau}_{1}\right], \\
0, & \text { otherwise }\end{cases} \\
& \chi_{2\left[t_{0}, t_{f}-\widehat{\tau}_{2}\right]}(t)= \begin{cases}1, & t \in\left[0, t_{f}-\widehat{\tau}_{2}\right], \\
0, & \text { otherwise. }\end{cases}
\end{aligned}
$$

By using the optimal conditions, (45) can be rewritten as follows:

$$
\begin{aligned}
& \frac{d \lambda_{1}}{d t} \\
& =-e^{-\delta t} \frac{a_{1} b_{1} E(t)}{\left(b_{1}+E(t) x(t)\right)^{2}} \\
& \quad+\lambda_{1}(t)\left[r\left(\frac{x(t)}{K}-1\right)+\frac{\alpha \beta y(t)}{(\alpha+x(t))^{2}}+E(t)\right]
\end{aligned}
$$




$$
\begin{aligned}
& \quad+\chi_{1\left[t_{0}, t_{f}-\widehat{\tau}_{1}\right]}(t) \frac{r x(t) \lambda_{2}\left(t+\widehat{\tau}_{1}\right)}{K} \\
& -\chi_{2\left[t_{0}, t_{f}-\widehat{\tau}_{2}\right]}(t) \frac{m \alpha \beta y(t)}{(\alpha+x(t))^{2}} \lambda_{2}\left(t+\widehat{\tau}_{2}\right), \\
& \frac{d \lambda_{2}}{d t} \\
& =-e^{-\delta t} \frac{a_{2} b_{2} E(t)}{\left(b_{2}+E(t) y(t)\right)^{2}}+\frac{\beta x(t) \lambda_{1}(t)}{\alpha+x(t)} \\
& +\lambda_{2}(t)\left(E(t)+d-\frac{m \beta x(t)}{\alpha+x(t)}\right) .
\end{aligned}
$$

In optimal control problem (40), there is not terminal cost and final state is free. Consequently, transversality conditions (bounded conditions) for adjoint functions are as follows: $\lambda_{k}\left(t_{f}\right)=0, k=1,2$.

It is easy to obtain characterization of optimal harvest control $E_{\delta}$, which derives that $\partial H /\left.\partial E\right|_{E=E_{\delta}}=0$. Consequently, it follows from simple computations that there exists an optimal harvest $E_{\delta}$ determined by

$$
\begin{aligned}
& \frac{a_{1} b_{1} x_{\delta}}{\left(b_{1}+E_{\delta} x_{\delta}\right)^{2}}+\frac{a_{2} b_{2} y_{\delta}}{\left(b_{2}+E_{\delta} y_{\delta}\right)^{2}} \\
& =c+\left[x_{\delta} \lambda_{1}(t)+y_{\delta} \lambda_{2}(t)\right] e^{\delta t},
\end{aligned}
$$

where $\lambda_{1}(t), \lambda_{2}(t)$ satisfies system (47) with $\lambda_{k}\left(t_{f}\right)=0, k=$ 1,2 .

Remark 8. It follows from similar arguments in Theorem 7 that symmetric optimal control problem on cases in Section 3.4 can be also obtained, which are omitted in this paper. $\tau_{1}$ and $\tau_{2}$ can be set to be a fixed value $\widehat{\tau}_{1} \in\left(0, \tau_{10}^{*}\right)$ and $\widehat{\tau}_{2} \in$ $\left(0, \tau_{2 w}\right)$, respectively; $\widehat{\tau}_{1} \neq \widehat{\tau}_{2}, \tau_{10}^{*}$, and $\tau_{2 w}$ are determined in Theorems 3 and 6 , respectively.

In the following part, the optimal system is numerically solved based on an iterative method with Runge-Kutta fourth order scheme. Firstly, by assuming that there exist a step size $\rho>0$ and integers $i, j, n$ with $\tau_{1}=i \rho, \tau_{2}=j \rho$, and $t_{f}-t_{0}=n \rho$. Secondly, we initialize optimal harvest control with a given initial value $E_{0}=0.09$. By using forward difference approximation, we solve the first and second differential equation of system (3) with initial conditions $x_{0}=0.38, y_{0}=$ 0.43 . Thirdly, by using backward difference approximation, we solve the adjoint functions (47) with transversality conditions. Finally, the optimal harvest control is updated by values of the state and adjoint variables, and the updated optimal harvest control is replaced with the initial value given in the second step. This process will be repeated until successive iterates of harvest control values become sufficiently close.

By utilizing combinations of forward and backward difference approximations, it can be derived that for $k=$ $0, \ldots, n-1$

$$
x_{k+1}=x_{k}+\rho\left[r x_{k}\left(1-\frac{x_{k-i}}{K}\right)-\frac{\beta x_{k} y_{k}}{\alpha+x_{k}}-E_{k} x_{k}\right],
$$

$$
\begin{aligned}
& y_{k+1}=y_{k}+\rho\left[y_{k}\left(\frac{m \beta x_{k-j}}{\alpha+x_{k-j}}-d\right)-E_{k} y_{k}\right], \\
& \lambda_{1}^{n-k-1}=\lambda_{1}^{n-k}-\rho e^{-\delta(n-k)} \frac{a_{1} b_{1} E_{k+1}}{\left(b_{1}+E_{k+1} x_{k+1}\right)^{2}} \\
& +\rho \lambda_{1}^{n-k}\left[r\left(\frac{x_{k+1}}{K}-1\right)+\frac{\alpha \beta y_{k+1}}{\left(\alpha+x_{k+1}\right)^{2}}+E_{k+1}\right] \\
& +\rho\left[\chi_{1\left[t_{0}, t_{f}-\widehat{\tau}_{1}\right]} \lambda_{1}^{n-k+i} \frac{r x_{k-j}}{K}\right. \\
& \left.-\chi_{2\left[t_{0}, t_{f}-\widehat{\tau}_{2}\right]} \lambda_{2}^{n-k+j} \frac{m \alpha \beta y_{k+1}}{\left(\alpha+x_{k+1}\right)^{2}}\right], \\
& \lambda_{2}^{n-k-1}=\lambda_{2}^{n-k}-\rho\left[e^{-\delta(n-k)} \frac{a_{2} b_{2} E_{k+1}}{\left(b_{2}+E_{k+1} y_{k+1}\right)^{2}}\right. \\
& \left.-\frac{\beta x_{k+1} \lambda_{1}^{n-k}}{\alpha+x_{k+1}}-\lambda_{2}^{n-k}\left(E_{k+1}+d-\frac{m \beta x_{k+1}}{\alpha+x_{k+1}}\right)\right] .
\end{aligned}
$$

In order to show effectiveness of optimal harvest control designed in Theorem 7, parameters values of system (3) are partially from [5], $r=2.5, K=1.4, m=0.6, \beta=1, \alpha=1.4$, $d=0.09, a_{1}=1, a_{2}=0.5, b_{1}=1.2, b_{2}=1, c=0.05, \delta=0.01$, $t_{0}=0$, and $t_{f}=120$ with appropriate units. By using given values of parameters and simple computations, interior equilibrium $P^{*}(0.2471,3.3911,0.7467)$ exists provided that $0<$ $v<0.1491$. In the following part, $v=0.075$ is utilized in the numerical simulations, which is arbitrarily selected within $(0,0.1491)$ and is enough to merit theoretical analysis obtained in this paper. It follows from Theorem 3, Theorem 7, and simple computations that system (3) is locally stable around $(0.2471,3.3911,0.7467)$ when $0<\tau_{1}<\tau_{10}^{*}=0.5632$ and $\tau_{2}=0$. By using similar arguments and Theorems 4 and 7 , it is revealed that system (3) is locally stable around ( 0.2471 , $3.3911,0.7467)$ when $0<\tau_{2}<\tau_{20}^{*}=0.3894$ and $\tau_{1}=0$. As analyzed in Section 3.3, $\widehat{\tau}_{2}=0.15$ is arbitrarily selected within $\left(0, \tau_{20}^{*}\right)$, that is, $(0,0.3894)$, and it follows from Theorems 5 and 7 that system (3) is locally stable around $(0.2471,3.3911$, $0.7467)$ when $0<\tau_{1}<\tau_{1 w}=0.4126$ and $\widehat{\tau}_{2}=0.15$. As analyzed in Section 3.4, $\widehat{\tau}_{1}=0.28$ is arbitrarily selected within $\left(0, \tau_{10}^{*}\right)$, that is, $(0,0.5632)$, and it follows from Theorems 6 and 7 that system (3) is locally stable around $(0.2471,3.3911$, $0.7467)$ when $\widehat{\tau}_{1}=0.28$ and $0<\tau_{2}<\tau_{2 w}=0.2219$. Numerical simulations are made to investigate dynamic effects of double time delays on variation of optimal prey, predator biomass, and optimal harvest control, which can be found in Figures 1 and 2, respectively. The initial values of simulation work made in Figure 1 are as follows: $x(0)=0.28, y(0)=$ $0.31, E(0)=0.76$, and initial values of simulation work made in Figure 2 are as follows: $x(0)=0.42, y(0)=0.21, E(0)=$ 0.81 . 

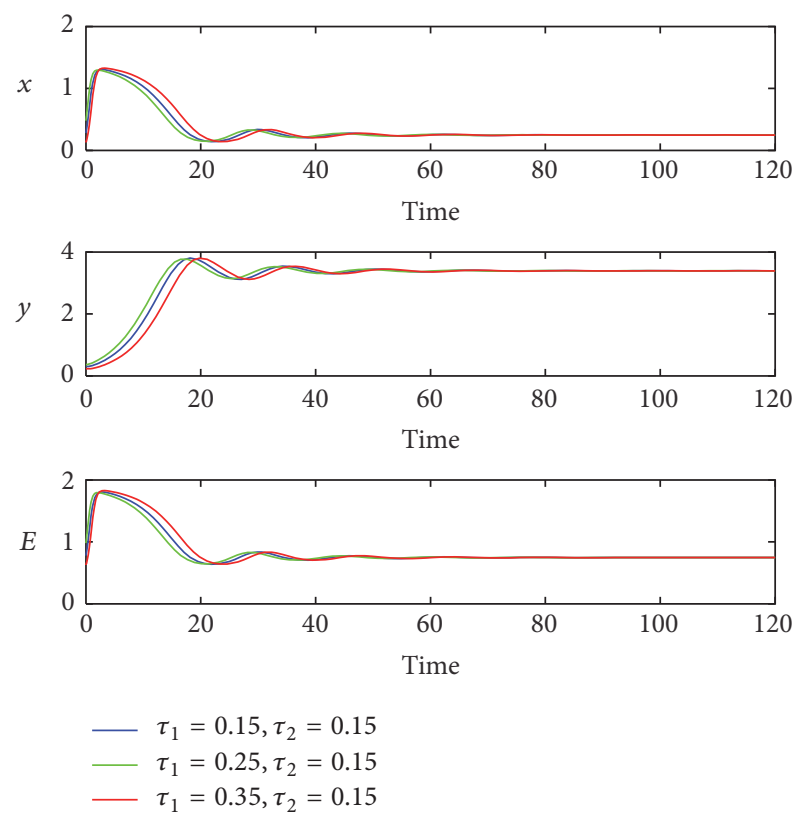

Figure 1: Stable dynamical variation of optimal prey, predator biomass and optimal harvest control with the increasing time under $\tau_{1}=0.15,0.25,0.35$ and $\tau_{2}=0.15$, respectively.
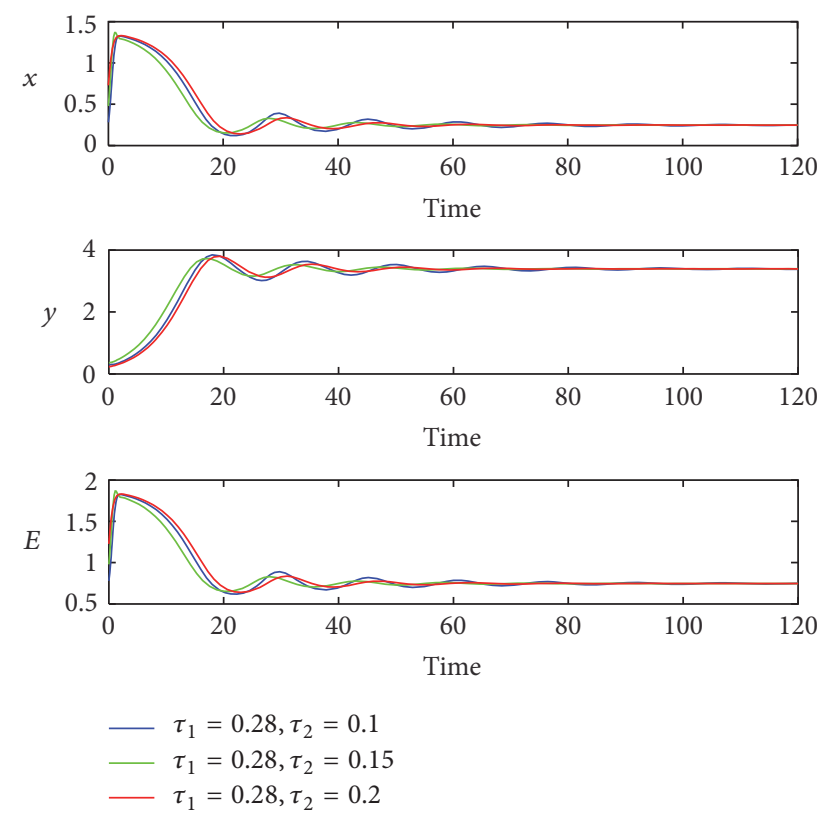

FIGURE 2: Stable dynamical variation of optimal prey, predator biomass and optimal harvest control with the increasing time under $\tau_{1}=0.28$ and $\tau_{2}=0.1,0.15,0.2$, respectively.

\section{Conclusion}

By incorporating maturation delay for prey population and gestation delay for predator population, we extend work done in [5]. In this paper, a singular prey-predator fishery model with double time delays is established. Fishing efforts are introduced to commercially harvest prey population and predator population, which are developed as control instruments to investigate optimal utilization of prey-predator fishery resource. Positivity of solutions and uniform persistence of system are discussed in Theorems 1 and 2. By analyzing associated characteristic transcendental equation, local stability around interior equilibrium is discussed due to combined variations of double time delays, which can be found in Theorem 3 to Theorem 6. Furthermore, an optimal harvest control is designed in Theorem 7. By using the proposed mathematical model and dynamical analysis, authors aim to obtain some results which are theoretically beneficial to discussing optimal harvesting strategies and sustainability mechanism of harvested prey-predator fishery system with double time delays. Furthermore, the theoretical results may be potentially constructive for administrative agencies to formulate regulatory policies to preserve economic optimality through ensuring global sustainability.

\section{Competing Interests}

All authors of this article declare that there is no conflict of interests regarding the publication of this article. We have no proprietary, financial, professional, or other personal interest of any nature or kind in any product, service, and/or company that could be construed as influencing the position presented in, or the review of, this article.

\section{Acknowledgments}

This work is supported by National Natural Science Foundation of China, Grant No. 61104003 and Grant No. 61673099, Research Program for Liaoning Excellent Talents in University, Grant No. LJQ2014027, and Hebei Province Natural Science Foundation, Grant No. F2015501047. This work is supported by Hong Kong Admission Scheme for Mainland Talents and Professionals, Hong Kong Special Administrative Region.

\section{References}

[1] Food and Agriculture Organization, The State of World Fisheries and Aquaculture 2014, FAO Corporate Document Repository, Food and Agriculture Organization of the United Nations, 2015.

[2] C. W. Clark, Mathematical Bioeconomics: The Optimal Management of Renewable Resource, John Wiley \& Sons, New York, NY, USA, 2nd edition, 1990.

[3] S. Lenhart and J. T. Workman, Optimal Control Applied to Biological Models, Chapman and Hall/CRC Mathematical and Computational Biology, Chapman and Hall/CRC, Boca Raton, Fla, USA, 2007.

[4] S. Behringer and T. Upmann, "Optimal harvesting of a spatial renewable resource," Journal of Economic Dynamics and Control, vol. 42, pp. 105-120, 2014.

[5] G.-I. Bischi, F. Lamantia, and D. Radi, "A prey-predator fishery model with endogenous switching of harvesting strategy," Applied Mathematics and Computation, vol. 219, no. 20, pp. 10123-10142, 2013.

[6] S. M. Bouguima and S. Benzerdjeb, "An age-structured fishery model: dynamics and optimal management with perfect elastic demand," Applied Mathematical Modelling, vol. 40, no. 1, pp. 218-232, 2016. 
[7] K. Chakraborty, S. Das, and T. K. Kar, "Optimal control of effort of a stage structured prey-predator fishery model with harvesting," Nonlinear Analysis. Real World Applications, vol. 12, no. 6, pp. 3452-3467, 2011.

[8] K. Chakraborty, S. Jana, and T. K. Kar, "Global dynamics and bifurcation in a stage structured prey-predator fishery model with harvesting," Applied Mathematics and Computation, vol. 218, no. 18, pp. 9271-9290, 2012.

[9] K. Chakraborty, S. Das, and T. K. Kar, "On non-selective harvesting of a multispecies fishery incorporating partial closure for the populations," Applied Mathematics and Computation, vol. 221, pp. 581-597, 2013.

[10] Y. F. Lv, R. Yuan, and Y. Z. Pei, "A prey predator model with harvesting for fishery resource with reserve area," Applied Mathematical Modelling, vol. 37, no. 5, pp. 3048-3062, 2013.

[11] D. Pal and G. S. Mahapatra, "A bioeconomic modeling of twoprey and one-predator fishery model with optimal harvesting policy through hybridization approach," Applied Mathematics and Computation, vol. 242, pp. 748-763, 2014.

[12] Y. Xue, S. Y. Tang, and J. H. Liang, "Optimal timing of interventions in fishery resource and pest management," Nonlinear Analysis. Real World Applications. An International Multidisciplinary Journal, vol. 13, no. 4, pp. 1630-1646, 2012.

[13] Y. Zhang, J. Li, Y. Jie, and X. Yan, "Optimal taxation policy for a prey-predator fishery model with reserves," Pacific Journal of Optimization, vol. 11, no. 1, pp. 137-155, 2015.

[14] H. S. Gordon, "The Economic theory of a common-property resource: the fishery," Journal of Political Economy, vol. 62, no. 2, pp. 124-142, 1954.

[15] C. Liu, N. Lu, Q. Zhang, J. Li, and P. Liu, "Modeling and analysis in a prey-predator system with commercial harvesting and double time delays," Applied Mathematics and Computation, vol. 281, pp. 77-101, 2016.

[16] A. F. Nindjin, M. A. Aziz-Alaoui, and M. Cadivel, "Analysis of a predator-prey model with modified Leslie-Gower and Hollingtype II schemes with time delay," Nonlinear Analysis: Real World Applications, vol. 7, no. 5, pp. 1104-1118, 2006.

[17] S. L. Yan, X. Z. Lian, W. M. Wang, and R. K. Upadhyay, "Spatiotemporal dynamics in a delayed diffusive predator model," Applied Mathematics and Computation, vol. 224, pp. 524-534, 2013.

[18] K. Gopalsamy, Stability and Oscillations in Delay Differential Equations of Population Dynamics, Kluwer Academic, London, UK, 1992.

[19] Y. Kuang, Delay Differential Equations with Applications in Population Dynamics, vol. 191, Academic Press, San Diego, Calif, USA, 1993.

[20] X. Yang, L. S. Chen, and J. F. Chen, "Permanence and positive periodic solution for the single-species nonautonomous delay diffusive models," Computers \& Mathematics with Applications, vol. 32, no. 4, pp. 109-116, 1996.

[21] H. R. Thieme, Mathematics in Population Biology, Princeton University Press, Princeton, NJ, USA, 2003.

[22] H. I. Freedman and V. S. H. Rao, "The trade-off between mutual interference and time lags in predator prey systems," Bulletin of Mathematical Biology, vol. 45, no. 6, pp. 991-1004, 1983. 


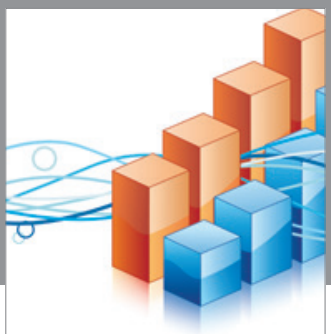

Advances in

Operations Research

vatem alat4

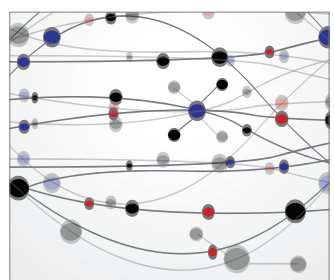

\section{The Scientific} World Journal
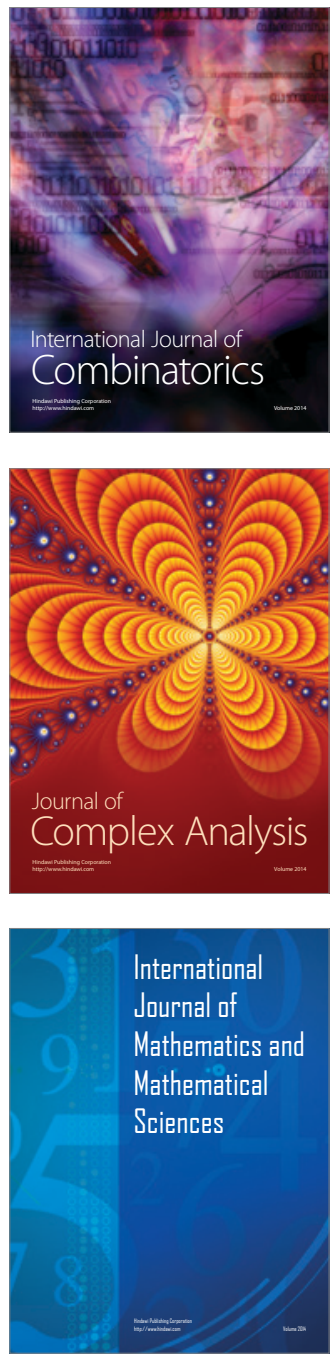
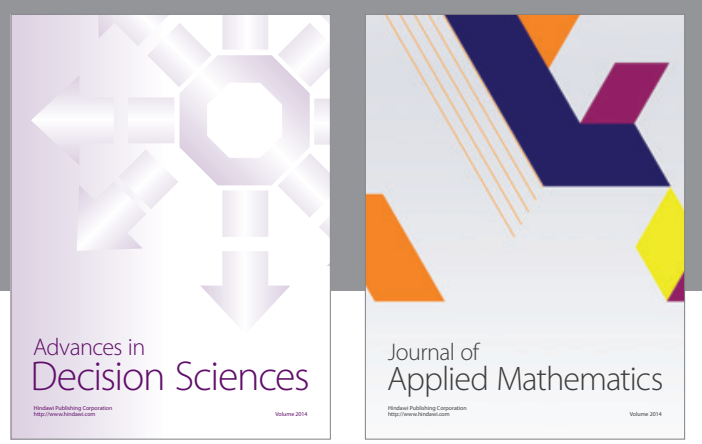

Algebra

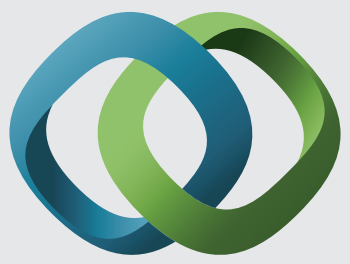

\section{Hindawi}

Submit your manuscripts at

http://www.hindawi.com
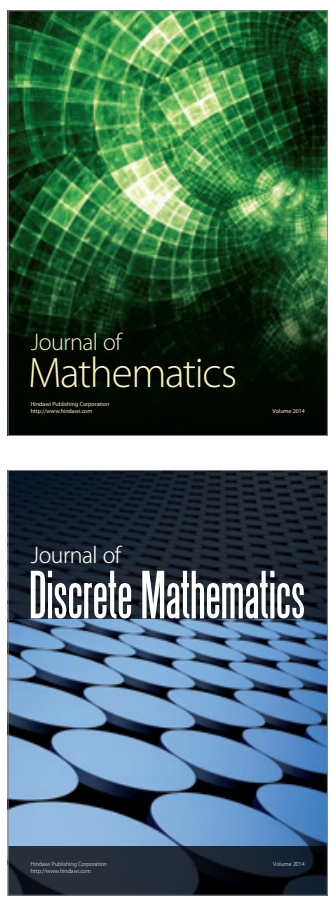

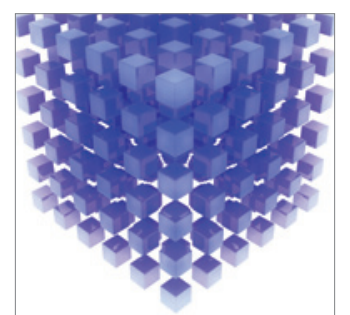

Mathematical Problems in Engineering
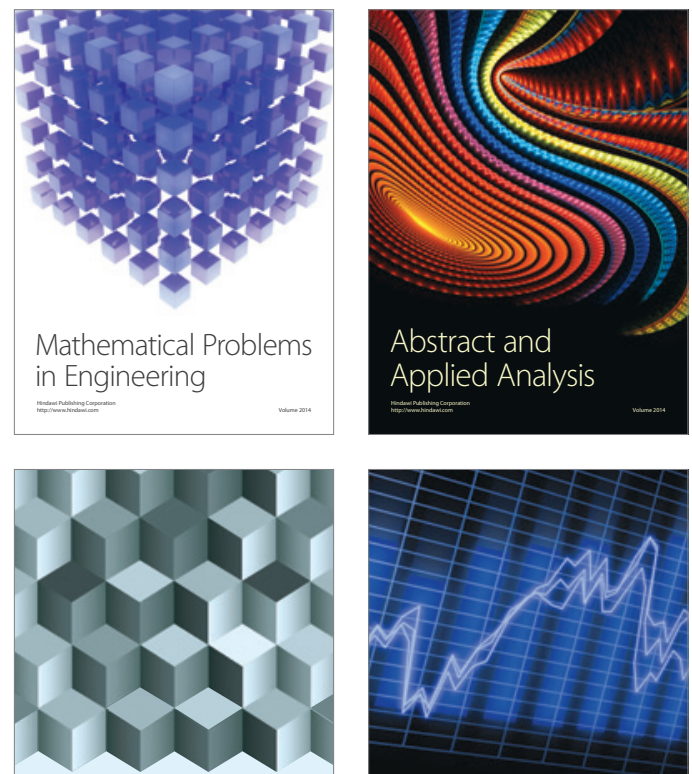

Journal of

Function Spaces

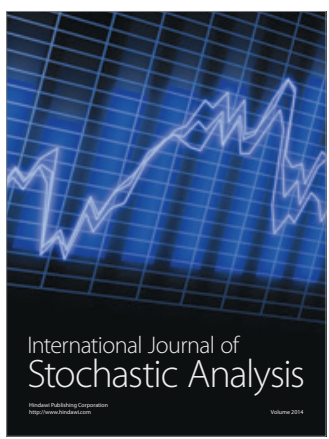

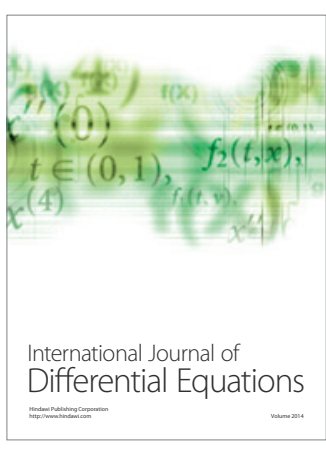
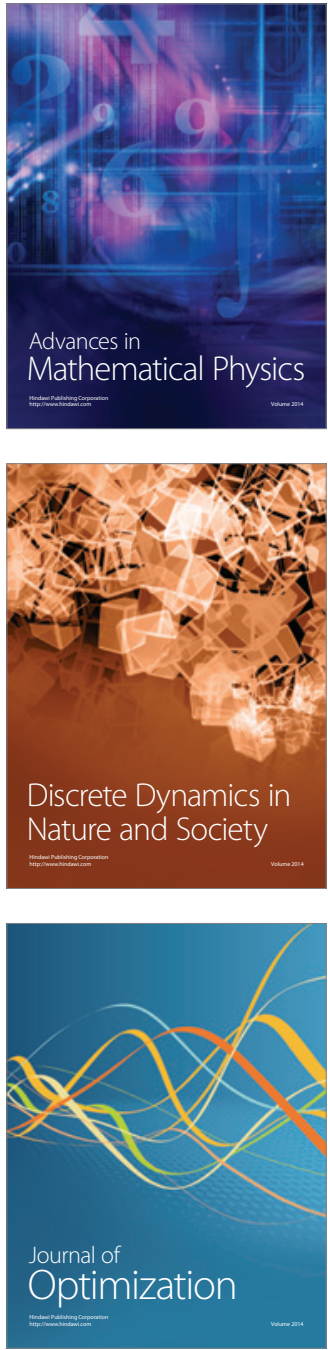